\title{
ESTUDO TERMODINÂMICO DE INCLUSÕES NÃO-METÁLICAS EM AÇO ACALMADO AO ALUMÍNIO E TRATADO COM CÁLCIO
}

Wagner Viana Bielefeldt ${ }^{1}$

Antônio Cezar Faria Vilela ${ }^{2}$

\section{Resumo}

O objetivo geral do trabalho foi o estudo da formação de inclusões não-metálicas no lingotamento contínuo (LC) para um aço acalmado ao alumínio e tratado com cálcio (SAE 8620). Os objetivos específicos foram: I) A obtenção das fases e compostos formados nas inclusões em função da composição e temperatura de lingotamento adotadas para 0 aço SAE 8620. 2) Estabelecer condições de composição química do aço para a formação de inclusões menos deletérias à lingotabilidade do SAE 8620. Com base em trabalhos da literatura e em trabalhos anteriores dos autores, simulações foram realizadas via FactSage e seus bancos de dados. As simulações foram realizadas utilizando a composição química global do aço SAE 8620 no distribuidor de LC. O resultado é a composição do aço e inclusões não-metálicas (óxidos e sulfetos) na temperatura de interesse. Os resultados mostraram a formação de diferentes óxidos sólidos e a formação de fase líquida nas inclusões pela variação do teor de cálcio no aço. Foi possível determinar a composição das inclusões como função dos teores de alumínio e cálcio no aço SAE 8620. Para uma determinada composição e temperatura do aço SAE 8620, também foi possível estabelecer uma faixa de teor de cálcio onde as inclusões são formadas por fase líquida predominante. Foi ainda possível calcular o percentual de fase líquida e sólida nas inclusões, bem como sua composição em termos de óxidos.

Palavras-chave: Inclusões; Tratamento com cálcio; FactSage.

\section{THERMODYNAMIC STUDY OF NON-METALLIC INCLUSIONS IN AL-KILLED CALCIUM TREATED STEEL}

\begin{abstract}
The main goal was the study of non-metallic inclusion formation in the continuous casting (CC) for Al-killed, calcium treated steel (SAE 8620). The specific goals were: I) To obtain the phases and compounds formed in the inclusions as a function of composition and casting temperature adopted for the SAE 8620 steel. 2) To establish conditions of steel chemical composition for the formation of less harmful inclusions to the SAE 8620 castability. Based in the literature and previous work of the authors, were carried out simulations using the software FactSage and databases. The simulations were realized using the global chemical composition of SAE 8620 steel in the CC tundish. The results were steel and non-metallic inclusions (oxides and sulphites) composition in the aimed temperature. The results showed the formation of different solid oxides and the formation of liquid phase in the inclusions by the variation of calcium content in the steel. It was possible to determine the inclusion composition as a function of aluminum and calcium content of SAE 8620 steel. For the specific composition and temperature of SAE 8620 steel, it was possible to establish a range of calcium content were the inclusions are formed by predominant liquid phase. It was possible to calculate the percent of liquid and solid phase in the inclusions, as well the composition in terms of oxides.
\end{abstract}

Key words: Inclusions; Calcium treatment; FactSage.

\footnotetext{
' Eng. Metalúrgico, Dr. Laboratório de Siderurgia/LaSid, Universidade Federal do Rio Grande do Sul - UFRGS, Av. Bento Gonçalves 9500 , Porto Alegre, RS, Brasil. E-mail: wagner@ct.ufrgs.br

2 Prof. Dr.-Ing. DEMET e PPGEM/UFRGS, Coordenador do Laboratório de Siderurgia/LaSid, Av. Bento Gonçalves 9500, Porto Alegre, RS, Brasil.

E-mail: vilela@ufrgs.br
} 


\section{INTRODUÇÃO}

Em aços acalmados ao alumínio, o cálcio é amplamente usado com o objetivo primário de modificar as inclusões sólidas de alumina para aluminatos de cálcio de menor ponto de fusão e maior deformabilidade. As adições de ligas a base de cálcio são utilizadas para controlar a composição, a distribuição e a morfologia das inclusões não-metálicas remanescentes no aço. $\bigcirc$ maior benefício desta prática está em minimizar a ocorrência de clogging na etapa de lingotamento contínuo (LC), ou seja, aumentar a sua lingotabilidade. Outros benefícios são: melhorias nas propriedades mecânicas dos aços, em geral, tenacidade e ductilidade, entre outros.

Vários fatores influenciam a lingotabilidade dos aços, por exemplo, os materiais refratários, a composição da escória, a cobertura do aço no distribuidor, o material da válvula e seu desenho, o controle de temperatura durante o processo, os tempos de espera etc. $\mathrm{O}$ controle de inclusões é somente um dos fatores. Entretanto, ele é muito importante na tentativa de melhorar a lingotabilidade.(I)

$\mathrm{Na}$ produção de aços especiais (como os destinados à indústria automotiva), os requisitos de qualidade são acima da média quando comparados com a maioria dos outros aços comerciais. Além disso, são aços críticos com relação à sua produção em aciaria, pois os teores de certos elementos no aço (Al, S, O) devem ser estritamente controlados. Então, conhecer o efeito das variações na composição química desses aços na formação e modificação de inclusões é fundamental. A termodinâmica computacional mostra-se como uma excelente ferramenta para a compreensão dos fenômenos físico-químicos que ocorrem em aciaria. A termodinâmica computacional pode também contribuir de forma decisiva nas tomadas de decisão dos engenheiros da planta industrial.

O objetivo geral deste trabalho foi o estudo termodinâmico da formação de inclusões não-metálicas no LC para o aço SAE 8620. Os objetivos específicos foram: I) A obtenção das fases e compostos formados nas inclusões em função da composição química e temperatura de LC adotadas para o aço SAE 8620. 2) Estabelecer condições de composição química do aço para a formação de inclusões menos deletérias à lingotabilidade do aço SAE 8620 .

\section{MATERIAIS E METODOLOGIA}

\section{I Materiais}

O aço SAE 8620 é classificado pela SAE-AISI como um aço níquel-cromo-molibdênio. Trata-se de um aço de baixa liga, usado em componentes que passam pelo processo de cementação. Segundo Silva e Mei, ${ }^{(2)} \circ$ aço SAE 8620 participa de uma série de aços para cemen- tação, os quais possuem temperabilidade suficiente para serem temperados em óleo, adquirindo, no núcleo, bons valores de ductilidade. São empregados para a construção de engrenagens, peças para trabalhos leves, pequenos mecanismos, pinos etc, materiais cuja característica mais importante é a resistência ao desgaste. A composição química utilizada foi obtida de uma série de corridas típicas de plantas industriais, e está indicada na Tabela I.

Tabela I. Composição química média para o aço SAE 8620 em \% em massa

\begin{tabular}{lcccccccccc}
\hline $\mathbf{C}$ & $\mathbf{S i}$ & $\mathbf{M n}$ & $\mathbf{S}$ & $\mathbf{A l}$ & $\mathbf{C a}$ & $\mathbf{C r}$ & $\mathbf{N i}$ & $\mathbf{M o}$ & $\mathbf{P}$ & $\mathbf{O}_{\text {total }}{ }^{*}$ \\
\hline 0,21 & 0,24 & 0,82 & 0,025 & 275,00 & $\mathrm{II}, 36$ & 0,47 & 0,47 & 0,16 & 0,017 & 18,4 \\
\hline *Al, $\mathrm{Ca}$ e $\mathrm{O}_{\text {total }}$ em ppm.
\end{tabular}

\subsection{Metodologia}

Com base em trabalhos anteriores dos autores, ${ }^{(3,4)}$ simulações termodinâmicas foram realizadas com o programa comercial FactSage e seus bancos de dados. $O$ FactSage foi introduzido em 200 I pela fusão dos pacotes termodinâmicos F*A*C*T/FACT-Win (Thermfact, Canadá) e ChemSage (GTT-Technology, Alemanha). As simulações foram realizadas utilizando a composição química global e temperatura média do aço SAE 8620 no distribuidor de $L C$ de $1.540^{\circ} \mathrm{C}$. O resultado obtido é a composição do aço e inclusões não-metálicas (óxidos e sulfetos) na temperatura de interesse, durante o LC.

O estudo termodinâmico foi realizado com o programa comercial FactSage, versão 6.2. Uma descrição geral do programa foi realizada por Bale et al.(5) Modificações recentes no FactSage e seus bancos de dados podem ser encontradas em Bale et al.(6)

Os seguintes bancos de dados foram utilizados, descritos a seguir segundo o FactSage Database Documentation:(7)

- FToxid - O banco de dados FToxid contém dados para sólidos e líquidos estequiométricos e soluções de óxidos de 20 elementos (bem como para soluções diluídas de $\mathrm{S}, \mathrm{SO}_{4}, \mathrm{PO}_{4}, \mathrm{H}_{2} \mathrm{O} / \mathrm{OH}, \mathrm{CO}_{3}$, $\mathrm{F}, \mathrm{Cl}, \mathrm{I}, \mathrm{C}, \mathrm{N}$ e CN em fase (escória) fundida. Dos diversos subsistemas de soluções no FToxid, foi utilizado o FToxid-SLAGA. Os principais componentes considerados nos cálculos foram: $\mathrm{FeO}$, $\mathrm{MnO}, \mathrm{SiO}_{2}, \mathrm{CaO}, \mathrm{Al}_{2} \mathrm{O}_{3}$ e $\mathrm{CaS}$;

- FSStel - Banco de dados para composições ricas em ferro. Dos diversos subsistemas no FSStel, utilizou-se a solução FSstel-LIQU.

Para o cálculo das janelas líquidas, foram utilizados os bancos de dados de soluções FToxid-SLAGA (para os óxidos e CaS) e o FSstel-LIQU (para o aço) no módulo Equilib do FactSage. 
Antes dos cálculos, os limites da janela (ou área) líquida foram determinados dos seguintes modos:

- pelo cálculo da linha de saturação de aluminatos de cálcio $\left(\mathrm{CaO} . \mathrm{Al}_{2} \mathrm{O}_{3}, \mathrm{CaO} .2 \mathrm{Al}_{2} \mathrm{O}_{3}\right)$, considerando um mínimo de $30 \%$ e $35 \%$ de $\mathrm{CaO}$ nas inclusões;

- pelo cálculo das linhas de saturação de CaS: máximo de $5 \%$ e $10 \%$ de $\mathrm{CaS}$.

Também uma análise mais detalhada da janela líquida foi realizada, levando-se em consideração as seguintes razões:

- seria interessante estabelecer, nas janelas líquidas, os percentuais de fase líquida e fase sólida das inclusões. Isso amplia o foco de estudo, não somente para lingotabilidade, mas também para um controle mais amplo da formação de fases nas inclusões;

- uma maior fração de sólidos nas inclusões de óxido pode auxiliar no controle da morfologia de sulfetos de manganês (MnS). Principalmente para evitar a formação de sulfetos alongados (tipo II), segundo Wakoh, Sawai e Mizoguchi;(8)

- segundo Fuhr e Cicutti(9) de acordo com resultados industriais, problemas de lingotabilidade ocorrem mais evidentemente quando a proporção de inclusões sólidas é maior que 60\%-70\%.

Considerando-se as razões apresentadas, uma abordagem diferente foi empregada. Utilizando-se somente os bancos de dados de soluções, não é possível calcular a formação ou não de fases sólidas. Na nova abordagem, foram usados nos cálculos, além dos bancos de dados de soluções, os sólidos puros estequiométricos (banco de dados FToxid do FactSage).

\section{RESULTADOS E DISCUSSÃO}

A Figura I apresenta a janela líquida, calculada usando-se a composição do aço $S A E 8620 \mathrm{e} \mathrm{T}=1.540^{\circ} \mathrm{C}$.

Para um teor de Al médio de 275 ppm (Tabela I), a janela de inclusões com um mínimo de $35 \%$ de $\mathrm{CaO}$ e um máximo de $5 \%$ de CaS está entre 10,6 ppm e 16,0 ppm de cálcio. O teor médio de cálcio apresentado na Tabela I (I I,36 ppm) indica que as corridas estão dentro da janela líquida.

A Figura 2 mostra a janela líquida do aço SAE 8620 e também: o início da formação de fase líquida, a formação de $40 \%$ de líquido e a formação de aproximadamente $100 \%$ de líquido, respectivamente.

$\mathrm{Na}$ Figura 2, a curva de $35 \%$ de $\mathrm{CaO}$ praticamente coincide com o início da formação de líquido. Entre 200 ppm e 400 ppm Al, é atingido 100\% de líquido na metade da janela - entre as curvas de $35 \%$ de $\mathrm{CaO}$ e $5 \%$ de CaS. Para 275 ppm de Al, têm-se inclusões com

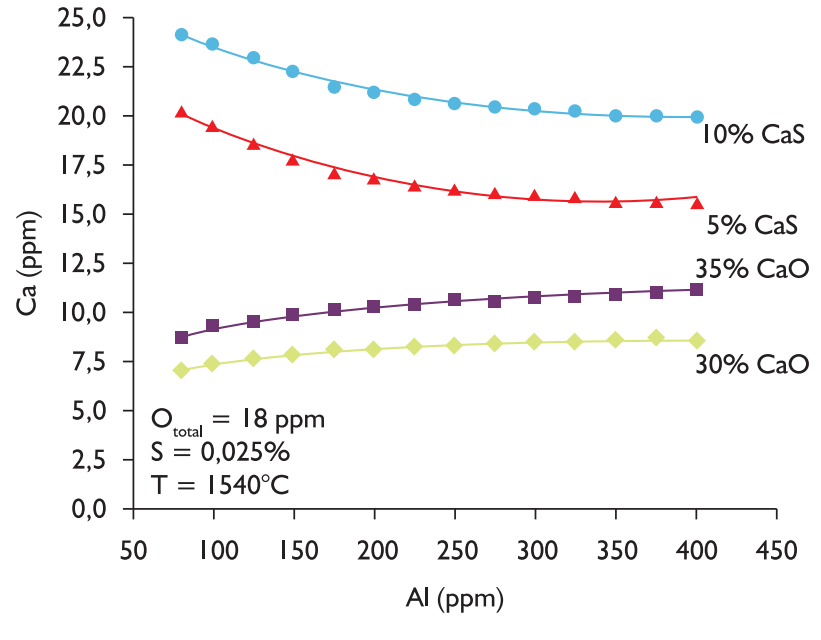

\begin{tabular}{|c|c|c|c|c|c|c|c|c|c|c|}
\hline & & \multicolumn{9}{|c|}{ Teor de Al, ppm } \\
\hline & & 80 & 100 & 150 & 200 & 250 & 275 & 300 & 350 & 400 \\
\hline $\min 30 \% \mathrm{CaO}$ & \multirow{4}{*}{ 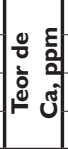 } & 7,00 & 7,40 & 7,90 & 8,20 & 8,30 & 8,40 & 8,50 & 8,50 & 8,60 \\
\hline $\min 35 \% \mathrm{CaO}$ & & 8,70 & 9,30 & 90 & 30 & 10,60 & 10,60 & 10,80 & 10,90 & 11,10 \\
\hline máx & & 20,20 & 19,50 & 17,70 & 16,70 & 16,20 & 16,00 & 15,90 & 15,60 & 15,50 \\
\hline máx $10 \% \mathrm{CaS}$ & & 24,10 & 23,60 & 22,20 & 21,10 & 20,60 & 20,40 & 20,30 & 20,00 & 19,90 \\
\hline
\end{tabular}

Figura I. Janela líquida para o aço SAE 8620.

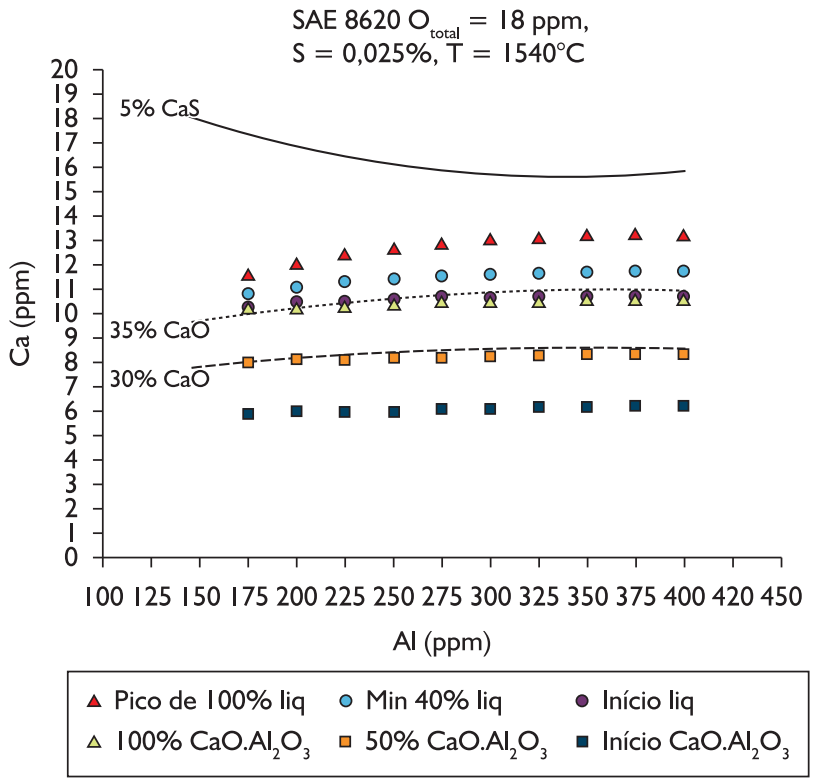

Figura 2. Janela líquida para o aço SAE 8620 , incluindo as curvas de formação de fração de líquido e de $\mathrm{CaO} \cdot \mathrm{Al}_{2} \mathrm{O}_{3}$.

$100 \%$ de fase líquida em aproximadamente 12,9 ppm de $\mathrm{Ca}$. Depois que se atinge cerca de $100 \%$ de líquido, esse percentual vai baixando proporcionalmente à formação de $\mathrm{CaS}$.

A Figura 2 inclui também o início da formação e

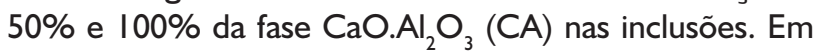
aços de $\mathrm{S}$ e $\mathrm{Al}$ elevados, muitas vezes não é possível a formação de inclusões líquidas sem a presença de $\mathrm{CaS}$. Segundo Kirsch-Racine, Bornont-Arzun e Contente, ${ }^{(10)}$ um nível de cálcio para garantir boa lingotabilidade e inclusões 
líquidas ou com alto teor de líquido está entre o CA e os óxidos líquidos. Gaye ${ }^{(I)}$ demonstra que, para um aço com $\mathrm{Al}=0,05 \%$ e $\mathrm{S}=0,035 \%$, o teor de $\mathrm{CaO}$ nas inclusões é limitado a $40 \%$ para evitar a formação de CaS. Inclusões de CA têm cerca de $35 \% \mathrm{CaO}$.

A Figura 3 foi calculada usando, além dos bancos de dados de soluções, os sólidos puros estequiométricos. A figura citada mostra o percentual em massa dos óxidos e $\mathrm{CaS}$ nas inclusões, dependendo do teor de cálcio total. A figura considera um nível fixo de alumínio total para o aço SAE 8620. Pode-se observar a modificação das inclusões de alumina pelo cálcio, resultando na seguinte sequência: $\mathrm{Al}_{2} \mathrm{O}_{3} \rightarrow \mathrm{CaO} .6 \mathrm{Al}_{2} \mathrm{O}_{3} \rightarrow \mathrm{CaO} .2 \mathrm{Al}_{2} \mathrm{O}_{3} \rightarrow \mathrm{CaO} . \mathrm{Al}_{2} \mathrm{O}_{3} \rightarrow$ óxidos líquidos $\rightarrow \mathrm{CaS}$. Isso está de acordo com o mecanismo de conversão de inclusões de $\mathrm{Al}_{2} \mathrm{O}_{3}$, proposto por Visser, Boom e Biglari. ${ }^{(12)}$

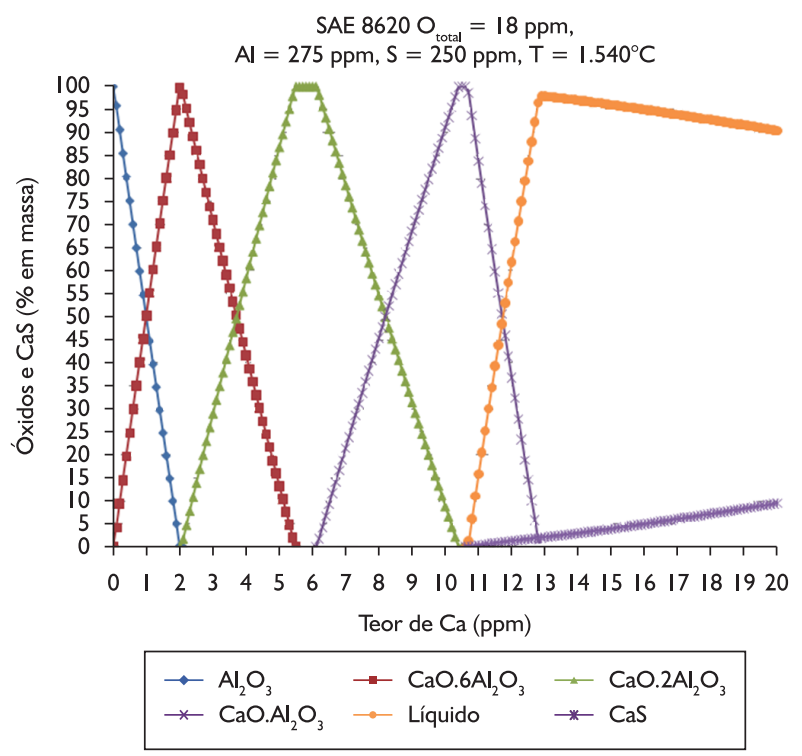

Figura 3. Percentual em massa dos óxidos e $\mathrm{CaS}$ nas inclusões $x$ teor de cálcio no aço SAE 8620 para $A \mathrm{l}=275$ ppm.

É possível comparar os resultados obtidos para o teor de cálcio entre a curva de $35 \% \mathrm{CaO}$ na Figura I, com a abordagem de determinação da formação de fase líquida nas inclusões. Existe pouca diferença entre as abordagens de cálculo. $O$ máximo seria próximo de $0,4 \mathrm{ppm}$ de cálcio para $\mathrm{Al}=400$ ppm (I I,7 ppm - I0,7 ppm). Na Figura I, $35 \%$ de $\mathrm{CaO}$ e $\mathrm{Al}=400 \mathrm{ppm}$ correspondem a II I I ppm Ca. A fase líquida inicia em 10,7 ppm Ca.

A Figura 4 mostra a composição da fase líquida considerando $\mathrm{Al}=275 \mathrm{ppm}$ e $\mathrm{O}_{\text {total }}=18 \mathrm{ppm}$ e demais condições para o aço SAE 8620. A fase líquida inicia-se com aproximadamente $38,4 \%$ de $\mathrm{CaO}$ e permanece praticamente constante até que não haja mais a formação de $\mathrm{CaO} . \mathrm{Al}_{2} \mathrm{O}_{3}$ (em I2,9 ppm Ca) (Figura 3). A partir daí, o teor de $\mathrm{Al}_{2} \mathrm{O}_{3}$ cai, enquanto o teor de $\mathrm{CaO}$ aumenta nas inclusões.

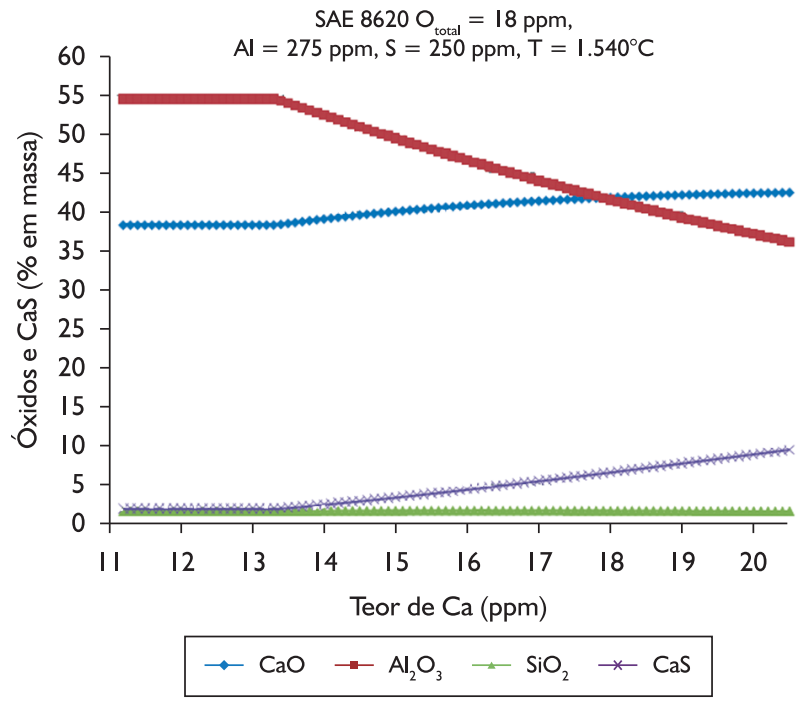

Figura 4. Composição da fase líquida para o aço SAE 8620, $\mathrm{Al}=275 \mathrm{ppm}$.

As Figuras 5 e 6 mostram a evolução dos teores de alumínio e cálcio dissolvidos no aço, dependendo do teor de cálcio total, para as condições do aço SAE 8620 $\left(\mathrm{Al}=275 \mathrm{ppm} \mathrm{e} \mathrm{O}_{\text {total }}=18 \mathrm{ppm}\right)$. As curvas apresentam uma inflexão cada vez que ocorre a formação de um dos óxidos. O alumínio dissolvido no aço vai aumentando conforme inclusões mais ricas em cálcio vão sendo formadas. O cálcio aumenta com o aumento do teor total de cálcio, porém o teor dissolvido é sempre muito baixo. O oxigênio dissolvido cai, como observado por Larsen e Fruehan. ${ }^{(13)}$ Considerando-se $\circ$ teor de cálcio total médio de II ppm (Tabela I), os valores obtidos foram: $\mathrm{Al}=255,3 \mathrm{ppm}$ (Figura 5), O = I,9 ppm e Ca $=0,6 \mathrm{I} \mathrm{ppm}$ (Figura 6 - onde lê-se \%Ca x 100 e \%O x I0).

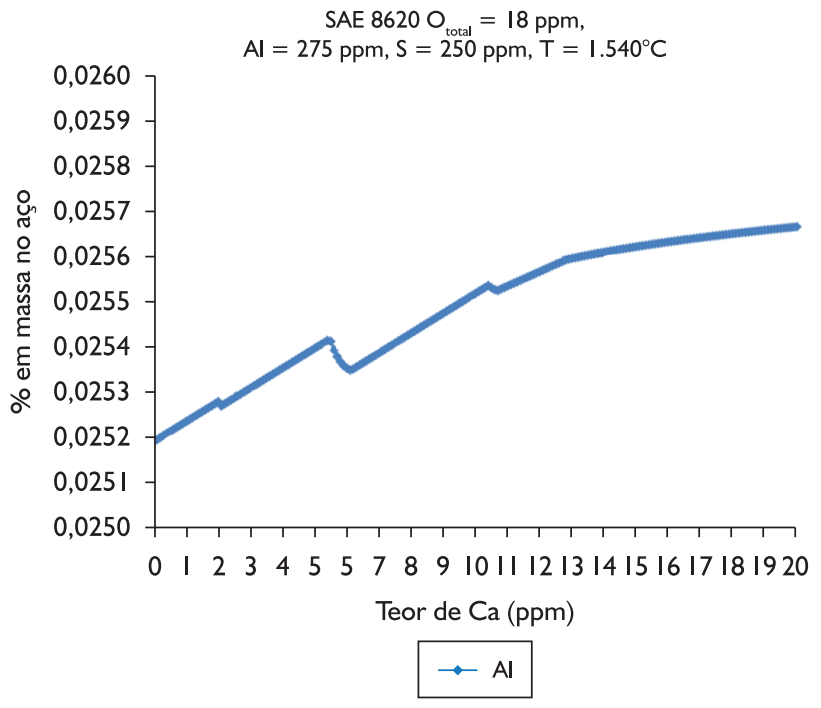

Figura 5. Teor de Al dissolvido dependendo do teor de Ca para o aço SAE 8620, $\mathrm{Al}=275$ ppm e $\mathrm{O}_{\text {total }}=18 \mathrm{ppm}$. 


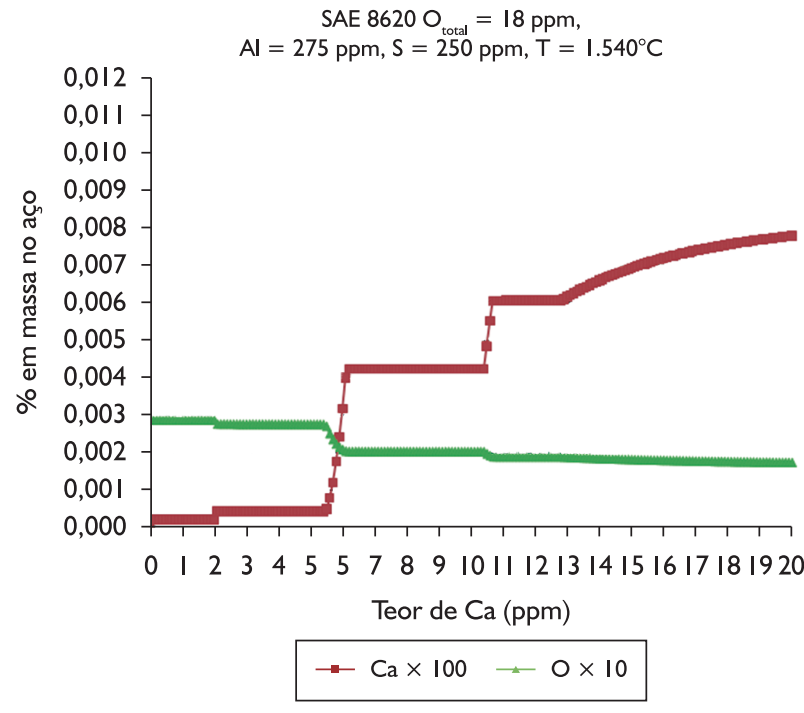

Figura 6. Teor de $\mathrm{Ca}$ e $\mathrm{O}$ dissolvido dependendo do teor de Ca para o aço $S A E 8620, \mathrm{Al}=275 \mathrm{ppm}$ e $\mathrm{O}_{\text {total }}=18 \mathrm{ppm}$.

As inclusões de $\mathrm{Al}_{2} \mathrm{O}_{3}$ modificadas pelo cálcio são frequentemente consideradas misturas binárias de $\mathrm{CaO}-\mathrm{Al}_{2} \mathrm{O}_{3}$. No entanto, as inclusões modificadas podem conter uma parcela significativa de $\mathrm{MgO}$. O MgO pode contribuir para $\circ$ sucesso da modificação com cálcio, de acordo com Pistorius, Presoly e Tshilombo.(14) $\mathrm{O}$ efeito do magnésio na formação de $\mathrm{MgO}$ e possíveis mudanças na janela líquida podem ser vistos no trabalho de Bielefeldt et al.(3)

\section{CONCLUSÕES}

Para um teor de $\mathrm{Al}$ médio de 275 ppm para o aço SAE 8620 , a $1.540^{\circ} \mathrm{C}$, a janela de inclusões com um mínimo de $35 \%$ de $\mathrm{CaO}$ e um máximo de $5 \%$ de $\mathrm{CaS}$ está entre 10,6 ppm e 16,0 ppm de cálcio. O teor médio de cálcio do aço SAE 8620 (I I,36 ppm) das corridas analisadas mostra que as mesmas estão dentro da janela líquida.

A curva de $35 \%$ de $\mathrm{CaO}$ da janela líquida do aço SAE 8620 praticamente coincide com o início da formação de fase líquida. Entre 200 ppm e 400 ppm de Al, é atingido $100 \%$ de líquido na metade da janela (entre as curvas de $35 \%$ de $\mathrm{CaO}$ e $5 \%$ de $\mathrm{CaS}$ ). Para 275 ppm de Al, têm-se inclusões com $100 \%$ de fase líquida em aproximadamente 12,9 ppm de Ca.

Pode-se constatar a modificação das inclusões de $\mathrm{Al}_{2} \mathrm{O}_{3}$ pelo cálcio, formando os diferentes aluminatos de cálcio, para diferentes teores de $\mathrm{Al}$ e $\mathrm{Ca}$ nas condições do aço SAE 8620.

Foi observada pouca diferença entre as abordagens de cálculo termodinâmico. $O$ máximo é próximo de 0,4 ppm de cálcio para $\mathrm{Al}=400 \mathrm{ppm}$, nas condições do aço SAE 8620.

Ainda para as condições do aço SAE 8620 apresentadas, o alumínio dissolvido no aço vai aumentando conforme inclusões mais ricas em cálcio vão sendo formadas. O cálcio aumenta com o aumento do teor de cálcio total, porém o teor dissolvido é sempre muito baixo. $O$ oxigênio dissolvido cai com o aumento do teor de cálcio total.

\section{Agradecimentos}

Wagner V. Bielefeldt agradece ao PNPD - MCT/ Finep, pela concessão de sua bolsa de pós-doutorado.

\section{REFERÊNCIAS}

I HOLAPPA, L. Inclusion control for castability of resulphurized steels. In: IRONMAKING CONFERENCE, 60., 200I, Baltimore, USA. Proceedings... (S.I.: s.n.), 200I. p. 1023-35.

2 SILVA, A. C; MEI, P. R. Aços e ligas especiais. 2. ed. São Paulo: Edgard Blücher, 2006.

3 BIELEFELDT, W. V. et al. Computational thermodynamics application on the calcium inclusion treatment of the SAE 8620 steel. Steel Research International, v. 78, n. 12, p. 857-62, Dec. 2007.

4 BIELEFELDT, W. V. Tratamento de inclusões não-metálicas com cálcio nos aços SAE I/4I e SAE 8620. 2009. I68 f. Tese (Doutorado em Engenharia de Minas, Metalúrgica e de Materiais) - Programa de Pós Graduação em Engenharia de Minas, Metalurgia e de Materiais da Universidade Federal do Rio Grande do Sul, Porto Alegre, 2009.

5 BALE, C. W. et al. FactSage thermochemical software and databases. Calphad, v. 26, n. 2, p. 189-228, June 2002.

6 BALE, C. W. et al. FactSage thermochemical software and databases: recent developments. Calphad, v. 33, n. 2, p. 295-3II, June 2009.

7 GTT Technologies. FactSage Database Documentation. Herzogenrath, 20 I0. [Parte integrante do programa FactSage versão 6.2].

8 WAKOH, M.; SAWAI, T.; MIZOGUCHI, S. Effect of S content on the MnS precipitation in steel with oxide nuclei. ISIJ International, v. 36, n. 8, p. I0I4-2I, 1996.

9 FUHR, F; CICUTTI, C. Relationship between nozzle deposits and inclusion composition in the continuous casting of steels. Iron and Steelmaker, v. 32, p. 53-8, Dec. 2003. 
10 KIRSCH-RACINE, A.; BORNONT-ARZUN, M.; CONTENTE, M. Calcium treatment of medium carbon steel grades for machinability enhancement: from the theory to industrial practice. Revue de Métallurgie-CIT, n. 12, p. 59I-7, Dec. 2007.

I I GAYE, H. Inclusion formation in steels. In: CRAMB, A. W.; BYRNE, M. The making, shaping and treating of steel-casting. Pittsburgh: The AISE Steel Foundation, 2003, p. I-20.

I2 VISSER, H.-J.; BOOM, R.; BIGLARI, M. Simulation of the Ca-treatment of Al-killed liquid steel. Revue de Métallurgie-CIT, n. 4, p. 172-80, Avr. 2008.

13 LARSEN, K.; FRUEHAN, R. J. Calcium modification of oxide inclusions. Transactions of the Iron \& Steel Society of AIME, v. 12, p. 125-32, Dec. 199I.

I4 PISTORIUS, P. C.; PRESOLY, P.; TSHILOMBO, K. G. Magnesium: origin and role in calcium treated inclusions. In: SOHN - INTERNATIONAL SYMPOSIUM ADVANCED PROCESSING OF METALS AND MATERIALS. v. 2. THERMO AND PHYSICOCHEMICAL PRINCIPLES: IRON AND STEEL MAKING. p. 373-378, TMS, 2006.

Recebido em: 10/03/20II

Aceito em: 05/04/20I I 